
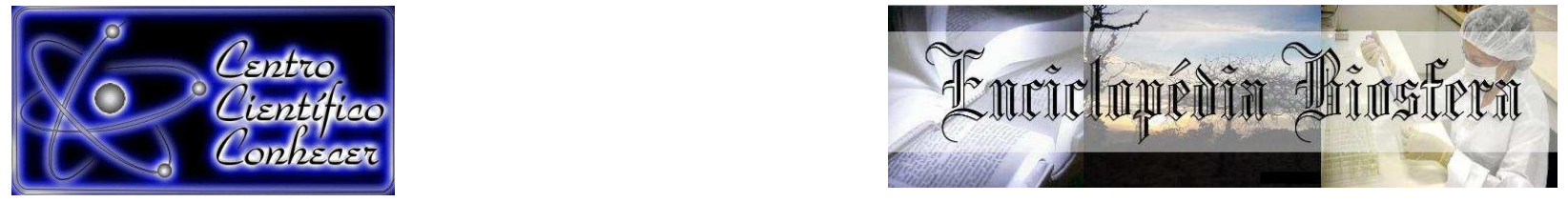

\title{
ATENDIMENTOS A IDOSOS REALIZADOS PELO SAMU EM MARINGÁ - PR NO ANO DE 2014
}

Aline Guzella Tokarski ${ }^{1}$, Adriana de Freitas Fantinelli ${ }^{2}$, Carla Kuniyosi Pellegrini ${ }^{3}$; Diógenes Aparício Garcia Cortez ${ }^{4}$; Lucia Elaine Ranieri Cortez ${ }^{5}$

1 Acadêmica do curso de Medicina do Centro Universitário de Maringá -

UNICESUMAR (lucia.cortez@unicesumar.edu.br) Maringá - Brasil

2 Acadêmica do curso de Medicina da Universidade Federal do Rio de JaneiroUFRJ

3 Acadêmico do curso de Medicina do Centro Universitário de Maringá UNICESUMAR

4 Professor do Mestrado em Promoção da Saúde do Centro Universitário de Maringá - UNICESUMAR e Pesquisador do Instituto Cesumar de Ciência, Tecnologia e Inovação (ICETI)

5 Professora do Curso de Medicina e Mestrado em Promoção da Saúde do Centro Universitário de Maringá - UNICESUMAR e Pesquisadora do Instituto Cesumar de Ciência, Tecnologia e Inovação (ICETI) Maringá - Brasil

Recebido em: 03/10/2016 - Aprovado em: 21/11/2016 - Publicado em: 05/12/2016 DOI: 10.18677/EnciBio_2016B_161

\begin{abstract}
RESUMO
O envelhecimento da população representa um dos maiores desafios da saúde pública nos dias de hoje, resultando em uma maior demanda na utilização de serviços médicos. O Serviço de Atendimento Móvel de Urgência (SAMU) é um serviço de atendimento pré-hospitalar móvel que tem como princípio básico o socorro imediato de vítimas e o encaminhamento ao serviço pré-hospitalar fixo ou hospitalar, diminuindo a mortalidade pelos agravos agudos, incluindo traumas. Este trabalho objetivou avaliar os atendimentos realizados pelo SAMU a população idosa de Maringá Paraná (PR). Trata-se de uma pesquisa descritiva, do tipo documental e retrospectivo, realizada a partir de dados secundários do SAMU de Maringá, PR. Foram analisados 956 prontuários de pacientes idosos atendidos pelo SAMU/Maringá-PR no período de junho a agosto de 2014. Entre os idosos 458 $(47,96 \%)$ eram do gênero feminino, sendo que a faixa etária de 70 a 79 anos foi a que mais necessitou dos serviços do SAMU/Maringá - PR. Os atendimentos foram mais frequentes no período vespertino $(31,79 \%)$ seguido do matutino $(31,58 \%)$. Dentre as causas relatadas evidenciou-se a dispneia e a hipertensão. Verificou-se que a maior parte dos idosos foram encaminhados para atendimento de média e alta complexidade. Estes resultados poderão auxiliar no desenvolvimento de estratégias de prevenção e promoção da saúde destes idosos, bem como para melhor atendimento destes pelo SAMU.
\end{abstract}

PALAVRAS-CHAVE: Assistência a Idosos; Serviços de Saúde para idosos; Serviços Médicos de Urgência. 


\title{
CARE FOR THE ELDERLY CONDUCTED BY SAMU IN MARINGÁ-PR
}

\begin{abstract}
The aging population is one of the greatest public health challenges today, resulting in a higher demand on the use of medical services. The Mobile Emergency Service (SAMU) is a mobile out-of-hospital care service that has as its basic principle the immediate relief of victims and their referral to the fixed out-of-hospital or hospital, reducing mortality by acute injuries, including trauma. This study aims to evaluate the care provided by SAMU the elderly (over 60 years) of Maringá -PR. This is a descriptive, documentary and retrospective type, made from secondary data from the SAMU, Paraná. We analyzed 956 medical records of elderly patients seen by the SAMU / Maringá-PR from June to August 2014. Among the 458 (47.96\%) were female, and the age group of 70 to 79 years was the most needed services SAMU / Maringá - PR. The calls were more frequent in the evening $(31.79 \%)$ followed by the morning (31.58\%). Among the reported causes was evident dyspnea and hypertension. It was found that most of the elderly were sent to service medium and high complexity. These results will assist in developing strategies for prevention and health promotion of these seniors, and for better care of this by the SAMU
\end{abstract}

KEYWORDS: Old Age Assistance; Health Services for the Aged; Emergengy Medical Services

\section{INTRODUÇÃO}

O envelhecimento da população representa um dos maiores desafios da saúde pública atualmente. Este fenômeno ocorreu inicialmente em países desenvolvidos, mas, recentemente, é nos países emergentes que o envelhecimento populacional tem ocorrido de forma mais acentuada e requerendo maior quantidade de pesquisas (BARBOSA \& NASCIMENTO, 2001; LIMA-COSTA \& VERAS, 2003; LIMA \& CAMPOS, 2011).

No Brasil, segundo pesquisa do IBGE, em 2011 a população idosa totalizou 23,5 milhões de pessoas e a projeção é que para 2020 sejam mais de 26 milhões de idosos no Brasil (DE MARCHI NETTO, 2006; OLIVEIRA et al., 2013; SILVA \&; SILVA, 2013; DANIACHI et al., 2015).

O Brasil tem experimentado mudanças no Sistema Único de Saúde desde a sua institucionalização em 1988, principalmente no tocante à regionalização e hierarquização dos serviços de saúde, no sentido de melhorar o atendimento aos usuários, entre eles, os que já passaram dos 60 anos e que estão frequentemente sujeitos a traumatismos e outras intercorrências emergenciais (MACHADO et al., 2011).

O aumento da população idosa resulta em novo perfil de morbimortalidade, sendo necessárias mudanças no acesso a serviços de saúde. O crescimento das doenças crônicas não-transmissíveis gera custos maiores ao Sistema Único de Saúde - SUS e trazem prejuízos a sociedade decorrente de mortes prematuras e incapacidades causadas. Ressaltando-se a importância de pesquisa, prevenção e promoção da saúde (BARBOSA et al., 2005).

Considera-se atendimento pré-hospitalar toda e qualquer assistência realizada, direta ou indiretamente, fora do âmbito hospitalar, utilizando-se meios e métodos disponíveis. Esse tipo de atendimento pode variar de um simples conselho ou orientação médica até o envio de uma viatura de suporte básico ou avançado ao local da ocorrência onde hajam pessoas traumatizadas, visando à manutenção da vida e à minimização de sequelas (MINAYO \& DESLANDES, 2008; MARQUES et 
al., 2011; VEGIAN \& MONTEIRO, 2011; VERONESE et al., 2012; GONSAGA et al., 2013).

A primeira etapa da implantação da Política Nacional de Atenção às Urgências ocorreu pela instituição do componente pré-hospitalar móvel, representado pelo SAMU 192, as Centrais de Regulação e seus Núcleos de Educação em Urgência (BRASIL, 2003). O serviço, que começou com recursos mínimos e área de abrangência restrita muitas vezes aos grandes centros, em 2014 já era composto por 3.092 unidades móveis em 182 Centrais de Regulação. As unidades são responsáveis pelo atendimento de 140,4 milhões de habitantes em 2.671 municípios brasileiros (BRASIL, 2014). Em estudo CABRAL \& SOUZA (2008) observaram que os idosos participam em torno de um terço das ocorrências por causas clínicas, prestadas pelo SAMU, ou seja, o Serviço Móvel de Atendimento faz parte do cotidiano desta população. Desta forma este artigo objetivou determinar os principais atendimentos realizados pelo SAMU a população idosa de Maringá no ano de 2014.

\section{MATERIAL E MÉTODOS}

Tratou-se de uma pesquisa descritiva do tipo documental e retrospectiva, realizada a partir de fontes secundárias no Serviço de Atendimento Móvel de Urgência de Maringá, Paraná (SAMU/Maringá - PR). De acordo com os preceitos éticos da Resolução: 196/96 do Ministério da Saúde que envolve pesquisa com seres humanos, foi garantido o anonimato dos pacientes quanto às informações coletadas. Obteve-se autorização institucional para uso do banco de dados do SAMU e aprovação do Comitê de Ética em Pesquisa da Unicesumar com CAAE n. 36779014700005539.

A cidade de Maringá - PR está situada no noroeste do estado Paraná, a 596 metros acima do nível do mar possui 473.064.190 $\mathrm{m}^{2}$ de superfície, uma população de 367.410 habitantes cuja parcela de moradores acima dos 60 anos é representada por 44.628 pessoas (BRASIL, 2012).

O levantamento das informações ocorreu na sede do SAMU, localizada na Avenida Prudente de Morais, 885 - Zona 07 do referido município. Este serviço público/municipal realiza assistência em situações de urgência clínica, traumática, psiquiátrica e obstétrica, em via pública, serviços de saúde ou domicílio, além do transporte de pacientes que necessitam de transferências interserviços de saúde.

Os dados foram coletados nos prontuários de pacientes atendidos pelo SAMU no período de agosto a setembro de 2014. Foi considerado critério de inclusão pacientes atendidos pelo SAMU com idade igual ou superior a 60 anos. Dessa forma, dos 2878 prontuários, 955 prontuários atenderam ao critério de inclusão.

Os dados foram analisados conforme dados socio-demográficos (sexo, idade) e as características do evento, tais como o mês, dia da semana, horário, gênero, faixa etária, tipo de causa clínica e externa, local de ocorrência e modalidade de encaminhamento.

Os dados obtidos foram digitados no programa Excel e importados para o software - Statistical Package for the Social Sciences - SPSS, -versão 12.0. Procedeu-se análise descritiva, por meio de frequências absolutas e percentuais. 


\section{RESULTADOS E DISCUSSÃO}

Dos 956 prontuários analisados $458(47,96 \%)$ foram do sexo feminino, 497 $(52,04 \%)$ masculino, e um $(0,10 \%)$ não estava indicado. Esse resultado é semelhante a outros estudos nacionais em relação ao maior atendimento para o sexo masculino, (GONSAGA et al., 2013; SILVA et al., 2013).

A Tabela 1 apresenta a faixa etária dos idosos atendidos pelo SAMU/Maringá/PR. Verificou-se maior percentual de idosos na faixa etária de 70-79 anos seguidos por 80 - 89 anos. Em estudo com adultos e idosos do município de Catanduva/SP foi verificado que aqueles com idade igual ou superior a 75 anos tiveram maior percentual de atendimentos (15,6\%) (GONSAGA et al., 2013). O envelhecimento resulta em alterações estruturais e funcionais que predispõem os idosos a diversos problemas de saúde, bem como a ocorrência de acidentes, que culminam na necessidade de atendimento do SAMU (PITTERI \& MONTEIRO, 2010).

TABELA 1 Estratificação de faixa etária dos atendimentos realizados pelo SAMU/Maringá-PR,- 2014 no período de agosto a setembro de 2014.

\begin{tabular}{lrrr}
\hline Faixa Etária & $\mathbf{N}$ & & $\%$ \\
\hline $\mathbf{6 0 - 6 9}$ & & 276 & 28,87 \\
$\mathbf{7 0 - 7 9}$ & 329 & 34,41 \\
$\mathbf{8 0 - 8 9}$ & 291 & & 30,44 \\
$\mathbf{9 0}$ ou mais & 60 & 6,28 \\
\hline Total & 956 & 100,00 \\
\hline
\end{tabular}

Já em relação aos dias da semana, a Tabela 2, evidencia que há uma pequena diminuição dos atendimentos na véspera e final de semana (sexta, sábado e domingo) em relação aos demais dias, destacando-se a sexta-feira com menor número de atendimentos 109 (11,40\%).

TABELA 2 Número de atendimentos realizados pelo SAMU/Maringá-PR em relação ao dia da semana, no período de agosto a setembro de 2014.

\begin{tabular}{|c|c|c|c|}
\hline Dias da Semana & $\mathbf{N}$ & & \\
\hline Dom & & 129 & 13,49 \\
\hline Seg & & 168 & 17,57 \\
\hline Ter & & 128 & 13,39 \\
\hline Qua & & 148 & 15,48 \\
\hline Qui & & 150 & 15,69 \\
\hline Sex & & 109 & 11,40 \\
\hline Sáb & & 124 & 12,97 \\
\hline Total & & 956 & 100,00 \\
\hline
\end{tabular}

O horário de atendimento com maior número de ocorrências foi o período vespertino, $12 \mathrm{~h}$ à $18 \mathrm{~h},(\mathrm{n}=304,31,79 \%)$ seguidos pelo matutino, 6h à $12 \mathrm{~h}(\mathrm{n}=302$, $31,58 \%)$ e período noturno ( $n=212,22,17 \%)$, como apresentado na Tabela 3. Resultados semelhantes também foram encontrados por GONSAGA et al. (2015), que relataram em estudo que a maioria das chamadas de atendimento de idosos para o SAMU, ocorrem no período diurno, em concordância com o período de maior atividade vigil. 
TABELA 3 Horário dos atendimentos realizados pelo SAMU/Maringá-PR, 2014, no período de agosto a setembro de 2014.

\begin{tabular}{|c|c|c|c|}
\hline Período & $\overline{\mathbf{N}}$ & & \\
\hline $10: 00 \mid---6^{6: 00}$ & & 109 & $\overline{11,4}$ \\
\hline 2 6:00|---12:00 & & 302 & 31,58 \\
\hline 3 12:00|- 18:00 & & 304 & 31,79 \\
\hline 4 18:00|- 24:00 & & 212 & 22,17 \\
\hline Não Registrados & & 29 & 3,03 \\
\hline Total & & 956 & 100,00 \\
\hline
\end{tabular}

Pode ser observado que o domicílio foi o local com maior número de solicitações do SAMU/Maringá-PR, seguido por unidades de saúde e via pública ( $\mathrm{n}=$ $668,18,83 \% ; n=180,18,83 \%$ e $n=56,5,86 \%$ ), respectivamente. A tabela 4 demonstra os principais motivos de acionamento do SAMU. A transferência destacase com um número de 132 solicitações (18,28\%).

Entre os principais motivos de causa clínica evidencia-se o sintoma dispneia $(n=71,9,83 \%)$, a Hipertensão Arterial Sistêmica (HAS) $(n=47,6,50 \%)$ e Diabetes Mellitus, $(n=29,4,01 \%)$, sendo que a HAS ocorre em maior frequência em idosos devido ao processo fisiológico de envelhecimento que predispõe o indivíduo a essas patologias. Na Hipertensão Arterial Sistêmica ocorrem alterações cardiocirculatórias, renais, e no mecanismo nervoso (MENDES \& BARATA, 2008). Já no Diabetes Mellitus há um aumento da resistência à insulina, diminuição da massa magra com aumento proporcional da massa adiposa, maior ingestão de carboidratos e polifarmácia (SENA et al., 2011).

Em outro estudo realizado em Porto Alegre/RS, as causas foram agrupadas por natureza do agravo ficando em ordem decrescente as neurológicas, cardiológicas e outros (MARQUES et al., 2011), sendo que as metodologias utilizadas podem justificar a diferença de frequências entre os estudos.

TABELA 4 Motivos de atendimento por causa clínica e transferência do SAMU/Maringá-PR no período de Junho a Agosto de 2014.

\begin{tabular}{lrr}
\hline \multicolumn{1}{c}{ Clínico } & N & $\%$ \\
\hline AVC & 26 & 3,61 \\
\hline Astenia & 20 & 2,77 \\
\hline Cardiopatia & 17 & 2,35 \\
\hline Convulsão & 25 & 3,46 \\
\hline Diabetes & 29 & 4,01 \\
\hline Dispnéia & 71 & 9,83 \\
\hline Dor precordial & 28 & 3,87 \\
Êmese & 26 & 3,61 \\
\hline Etilismo & 15 & 2,07 \\
HAS & 47 & 6,50 \\
Outros & 175 & 24,23 \\
Psiquiátrico & 16 & 2,21 \\
RCP-USA & 14 & 1,93 \\
Rebaixamento de Consciência & 38 & 5,26 \\
\hline Sem Registro & 17 & 2,35 \\
\hline Síncope & 26 & 3,61 \\
\hline Transferência & 132 & 18,28 \\
\hline \multicolumn{1}{|c|}{ Total } & 722 & 100,00 \\
\hline ENCICLOPÉDIA BIOSFERA, Centro Científico Conhecer - Goiânia, v.13 n.24; p.1757 & 2016 \\
\hline
\end{tabular}


A Unidade de Pronto Atendimento (UPA) Zona Norte (29,77\%) foi o local mais freqüente de encaminhamentos para os atendimentos por causas clínicas, seguida da UPA Zona Sul $(19,25 \%)$, correspondendo juntas a quase metade dos locais de destino $(49,02 \%)$, conforme visualizado na tabela 5 .

TABELA 5 Local de encaminhamento dos atendimentos por causa clínica do SAMU/Maringá-PR no período de Junho a Agosto de 2014.

\begin{tabular}{lrr}
\hline Clínico Destino & N & \multicolumn{2}{c}{$\%$} \\
\hline H.C. & 7 & 0,96 \\
HUM & 69 & 9,55 \\
Memorial & 11 & 1,52 \\
Metropolitano & 34 & 4,70 \\
Municipal & 36 & 4,98 \\
Outros & 25 & 3,46 \\
Santa Rita & 105 & 14,54 \\
Sem Encaminhamento & 26 & 3,60 \\
Santa Casa & 55 & 7,61 \\
UPA Zona Norte & 215 & 29,77 \\
UPA Zona Sul & 139 & 19,25 \\
\hline Total & 722 & 100,00 \\
\hline
\end{tabular}

Entre as causas externas de atendimento do SAMU/Maringá-PR a queda de mesmo nível foi o principal motivo, representando $62,50 \%$ dos atendimentos (tabela 6). Tal achado corrobora com estudo realizado em João Pessoa/PB em que $74,4 \%$ das causas externas de solicitação do atendimento do SAMU foram devido a quedas (OLIVEIRA et al., 2013).

A longevidade predispõe os indivíduos a um maior número de patologias como problemas mentais, cárdio-cerebrovasculares, oncológicos, articulares, dentre outras, necessitando utilização de inúmeros medicamentos. Estes podem repercutir na mobilidade, dependência física e mental, favorecendo episódios de queda (GILBERT et al., 2012). Em estudo REIS et al., (2015) relatam que idosos com problemas mentais apresentam uma elevada prevalência de quedas, sendo muitos os fatores de risco, ressaltando a idade avançada e doença mental.

TABELA 6 Causas externas de atendimento do SAMU/Maringá-PR no período de Junho a Agosto de 2014.

\begin{tabular}{llr}
\hline Causas Externas & N & \multicolumn{2}{c}{$\%$} \\
\hline Acidente de Trânsito & 3 & 3,75 \\
Afogamento & 1 & 1,25 \\
Amputação de Dedo & 1 & 1,25 \\
Asfixia por Alimento & 1 & 1,25 \\
Asfixia: Engasgo & 1 & 1,25 \\
Choque Anaflático & 1 & 1,25 \\
Complicação Pós- operatória & 2 & 2,50 \\
Ferimento Corto-contuso & 4 & 5,00 \\
Intoxicação & 1 & 1,25 \\
Mal estar Pós-exame & 1 & 1,25 \\
Picada de Cobra & 1 & 1,25 \\
\hline
\end{tabular}




\begin{tabular}{lrr}
\hline Queda de Cadeira & 6 & 7,50 \\
Queda de Mesmo Nível & 50 & 62,50 \\
Queda de Nível Elevado & 2 & 2,50 \\
Queda de Ónibus & 1 & 1,25 \\
Reação Alérgica & 1 & 1,25 \\
Suicídio & 1 & 1,25 \\
Trauma ao Sentar & 1 & 1,25 \\
Trauma Genital & 1 & 1,25 \\
\hline Total & 80 & 100,00 \\
\hline
\end{tabular}

O local de encaminhamento dos atendimentos devido a causa externa realizado pelo SAMU/Maringá-PR foi o Hospital Universitário de Maringá (HUM) $(42,50 \%)$ (tabela 7$)$. Diferentemente do observado no encaminhamento de causas clínicas, as causas externas concentraram-se no Hospital Universitário Regional de Maringá (HUM) sugerindo que esse tipo de acometimento necessite de atendimento de nível maior de complexidade.

TABELA 7 Local de encaminhamento dos atendimentos por causa clínica do SAMU/Maringá-PR no período de Junho a Agosto de 2014.

\begin{tabular}{lrr}
\hline Causas Externas Destino & N & \multicolumn{2}{c}{$\%$} \\
\hline HUM & 34 & 42,50 \\
Metropolitano & 6 & 7,50 \\
Santa Casa & 3 & 3,75 \\
Santa Rita & 15 & 18,75 \\
Sem Registro & 3 & 3,75 \\
UPA Zona Norte & 11 & 13,75 \\
UPA Zona Sul & 8 & 10,00 \\
\hline Total & 80 & 100,00 \\
\hline
\end{tabular}

\section{CONCLUSÕES}

Os idosos são uma parcela da população que solicitam uma grande parte dos atendimentos do SAMU e a maioria dos mesmos são decorrentes de patologias favorecidas pela longevidade.

Ressalta-se que com o aumento da população idosa, deverá ocorrer uma crescente procura por este serviço pelos mesmos e a importância do desenvolvimento de estratégias de prevenção e promoção da saúde para os idosos, bem como um melhor atendimento destes pelo SAMU.

\section{REFERÊNCIAS}

BARBOSA, M.L.J.; NASCIMENTO, E.F.A. Incidência de internações de idosos por motivos de quedas em um hospital geral de Taubaté. Revista Biociência, Taubaté, v.7, n.1, p.35-42, 2001. Disponível em: http://periodicos.unitau.br/ojs2.2/index.php/biociencias/article/view/76

BARBOSA, A.R; ZOUZA, J. M. P.; LEBRÃO, M. L.; RUY, L.; MARUCCI, M. F. Antropometria em idosos no município de São Paulo, Brasil. Cad Saude Publica v.21, n.6, p 1929-1938, 2005. 
BRASIL. Ministério da Saúde. Portaria 1864/ GM de 29 de Setembro de 2003. Institui o componente pré-hospitalar móvel da Política Nacional de Atenção às Urgências, por intermédio da implantação de Serviços de Atendimento Móvel de Urgência em municípios e regiões de todo o território brasileiro: SAMU- 192. Diário Oficial da União, Brasília, DF, 6 out. 2003.

BRASIL. Ministério da Saúde. DATASUS - 2012. Disponível em $<$ http://tabnet.datasus.gov.br/cgi/ deftohtm.exe?ibge/cnv/popbr.def>. Acesso em 14 maio 2015.

BRASIL. Ministério da Saúde. Coordenação de Amanda Costa e Vera Stumm. Desenvolvido pela ASCOM / MS, 2014. Apresenta textos sobre o Sistema Único de Saúde. Disponível em <http://portalsaude.saude.gov.br/index.php/cidadao/principal/agencia-saude>. Acesso em: 9 maio 2015.

CABRAL, A. P. S.; SOUZA, W. V. Serviço de Atendimento Móvel de Urgência (SAMU): análise da demanda e sua distribuição espacial em uma cidade do Nordeste brasileiro. Rev. Bras. Epidemiol., São Paulo, v. 11, n. 4, p.530-540, 2008 . Disponível em: . Acesso em: 20 maio 2015.

DANIACHI, D.; NETTO, A. S.; ONO, N. K.; GUIMARÃES, R. P.; POLESELLO, G. C.; HONDA, E. K. Epidemiologia das fraturas do terço proximal do fêmur em pacientes idosos. Revista Brasileira de Ortopedia, n.4, v.50, p. 371-377, 2015. Disponível em: < http://dx.doi.org/10.1016/j.rbo.2014.07.014> doi 10.1016/j.rbo.2014.07.014.

DE MARCHI NETTO, F.L. Aspectos biológicos e fisiológicos do envelhecimento humano e suas implicações na saúde do idoso. Pensar a prática, v. 7, n. 1, p. 7584, 2006. Disponível em: <http://dx.doi.org/10.5216/rpp.v7i1.67> doi 10.5216/rpp.v7i1.67

GILBERT, T., SZCZERBIN'SKA, K., ZIERE, G., BONNEFOY, M., VAN DER VELDE, N., CRUZ-JENTO, A. (2012). Management of falls: France Netherland, Poland. $\begin{array}{llll}\text { European } \quad \text { Geriatric } & \text { Medicine, } & \end{array}$ doi:10.1016/j.eurger.2012.05.001

GONSAGA, R. A. T.; BRUGUGNOLLI, I. D.; ZANUTTO, T. A.; GILIOLI, J. P.; SILVA, L. F. C.; FRAGA, G. P. Características dos atendimentos realizados pelo Serviço de Atendimento Móvel de Urgência no município de Catanduva, Estado de São Paulo, Brasil, 2006 a 2012. Epidemiologia e Serviços de Saúde, v. 22, n. 2, p. 317-324, 2013. Disponível em: <http://dx.doi.org/10.5123/S1679-49742013000200013> doi: 10.5123/S1679-49742013000200013.

GONSAGA, R. A. T.; DA SILVA, E. M.; BRUGUGNOLLI, I., D.; CABRAL, J. L.alini Cabral; NETO O. T.. Padrão e necessidades de atendimento pré-hospitalar a idosos. Rev. Bras. Geriatr. Gerontol., Rio de Janeiro, n. 18, v.1, p.19-28, 2015.

LIMA, R.S.; CAMPOS, M.L.P. Perfil do idoso vítima de trauma atendido em uma Unidade de Urgência e Emergência. Revista Escola de Enfermagem da USP, São 
Paulo, v.45, n.3, p. 659-664, 2011. Disponível em: <http://dx.doi.org/10.1590/S008062342011000300016> doi 10.1590/S0080-62342011000300016.

LIMA-COSTA, M.F.; VERAS, R. Saúde Pública e envelhecimento. Revista Caderno de Saúde Pública, Rio de Janeiro, vol.19, n.3, p 700-701, 2003. Disponível em: <http://dx.doi.org/10.1590/S0102-311X2003000300001> doi 10.1590/S0102$311 \times 2003000300001$

MACHADO, C.V.; SALVADOR, F.G.F.; O'DWYER, G. Serviço de Atendimento Móvel de Urgência: análise da política brasileira. Revista Saúde Pública, Rio de Janeiro, v.45, n.3, p.519-528, 2011. Disponível em: <http://dx.doi.org/10.1590/S0034$89102011005000022>$ doi 10.1590/S0034-89102011005000022.

MARQUES, G.Q.; LIMA, M. A. D. S.; CICONET, R.M. Agravos clínicos atendidos pelo serviço de atendimento móvel de urgência (SAMU) de Porto Alegre-RS. Acta Paul Enferm, v. 24, n. 2, p. 185-91, 2011. Disponível em: < http://dx.doi.org/10.1590/S0103-21002011000200005 > doi 10.1590/S010321002011000200005.

MENDES, R.; BARATA, J.L.T. Envelhecimento e Pressão arterial. Acta Med Port, p.193-198, 2008.

MINAYO, M.C.S.; DESLANDES, S.F. Análise da implantação do sistema de atendimento pré-hospitalar móvel em cinco capitais brasileiras. Revista Caderno de Saúde Pública, Rio de Janeiro, v. 24, n.8, p 1877-1886, 2008. Disponível em: <http://dx.doi.org/10.1590/S0102-311X2008000800016> doi 10.1590/S0102311 X2008000800016.

OLIVEIRA, F. M. R. L. de et al. Caracterização do trauma em idosos atendidos em serviço de atendimento móvel de urgência.Revista da Rede de Enfermagem do Nordeste-Rev Rene, v. 14, n. 5, 2013. Disponível em: http://www.revistarene.ufc.br/revista/index.php/revista/article/view/1344/pdf_1

PITTERI, J.S.M.; MONTEIRO, P. S. Caracterização do Serviço de Atendimento Móvel de Urgência (SAMU) em Palmas- Tocantins, Brasil, em 2009. Comun Ciênc Saúde n.21, v.3, p.227-36, 2010.

REIS, G.; MAYOR, M. S.; PESTANA, H.; SANTOS, J. M. Quedas, idade avançada e doença mental. RIASE, v.1, n.3, p.323-337, 2015.

SENA, I.B.; NOBRE, J.R.S.; COMPARIN, K.A. Envelhecimento e diabetes: a percepção do diabético tipo II. FIEP BULLETIN, v. 81, Special Edition - Article II, $2011 . \quad$ Disponível em: http://www.fiepbulletin.net/index.php/fiepbulletin/article/viewFile/276/500

SILVA, A.P.F.; SILVA, L.L. Perfil epidemiológico dos idosos atendidos pelo serviço de atendimento móvel de urgência (SAMU) na cidade de Maceió/Al. Caderno de Graduação-Ciências Biológicas e da Saúde-FITS, v. 1, n. 2, p. 135-143, 2013. 
VEGIAN, C.F.L.; MONTEIRO, M.I. Condições de vida e trabalho de profissionais de um Serviço de Atendimento Móvel de Urgência. Rev. Latino-Am. Enfermagem, Ribeirão Preto , v. 19, n. 4, p. 1018-1024, Aug. 2011 . Disponivel em: <http://dx.doi.org/10.1590/S0104-11692011000400022> doi 10.1590/S010411692011000400022.

VERONESE, A.M.; OLIVEIRA, D.L.L.C.; NAST, C. Risco de vida e natureza do SAMU: Demanda não pertinente e implicações para a enfermagem. Revista Gaúcha de Enfermagem, Porto Alegre, v.33, n.4, p. 142-148, 2012. Disponivel em: <http://dx.doi.org/10.1590/S1983-14472012000400018>. doi 10.1590/S198314472012000400018. 